\title{
Ultra-Wideband, Short-Pulse Ground-Penetrating Radar: Simulation and Measurement
}

\author{
Stanislav Vitebskiy, Student Member, IEEE, Lawrence Carin, Senior Member, IEEE, \\ Marc A. Ressler, Member, IEEE, and Francis H. Le
}

\begin{abstract}
Ultra-wideband (UWB), short-pulse (SP) radar is investigated theoretically and experimentally for the detection and identification of targets buried in and placed atop soil. The calculations are performed using a rigorous, three-dimensional (3-D) Method of Moments algorithm for perfectly conducting bodies of revolution. Particular targets investigated theoretically include anti-personnel mines, anti-tank mines, and a 55-gallon drum, for which we model the time-domain scattered fields and the buried-target late-time resonant frequencies. With regard to the latter, the computed resonant frequencies are utilized to assess the feasibility of resonance-based buried-target identification for this class of targets. The measurements are performed using a novel UWB, SP synthetic aperture radar (SAR) implemented on a mobile boom. Experimental and theoretical results are compared.
\end{abstract}

\section{INTRODUCTION}

$\mathbf{T}$ HE INSIDIOUS nature of mines has stimulated significant research-spanning over more than half a century-on techniques for buried-mine detection and identification. A significant tool for such is ground penetrating radar [1]-[18]. The frequencies used in GPR are chosen such that good ground penetration can be achieved, which necessitates relatively low operating frequencies $(<1 \mathrm{GHz})$ for the losses common in typical soils [19]-[21]. Further, the bandwidth must be as large as possible to achieve sufficient temporal (spatial) resolution. With these motivations, ultra-wideband (UWB), short-pulse (SP) systems have received significant interest recently [22], [23]. Many of these systems operate in the time domain and have an instantaneous frequency spectrum of approximately $0.1-1.0 \mathrm{GHz}$ (>100\% bandwidth).

We have developed new three-dimensional (3-D) numerical algorithms for the modeling of UWB-SP scattering from realistic targets placed atop and buried in lossy, dispersive soils. Our Method of Moments (MoM) algorithm is implemented in the frequency domain, thereby allowing the modeling of dispersive soils [15]-[18]. Although the targets must be bodies of revolution, most practical targets of interest (buried mines, canisters, etc.) can be modeled. Our current code [16]-[18] assumes that the target is perfectly conducting, and the soil is modeled as a lossy, dispersive half space.

To use the MoM, one requires the accurate and efficient computation of the half-space dyadic Green's function over

Manuscript received April 29, 1996; revised October 15, 1996.

S. Vitebskiy and L. Carin are with the Department of Electrical and Computer Engineering, Duke University, Durham, NC 27708-0291 USA (email: lcarin@ee.duke.edu).

M. A. Ressler and F. H. Le are with the Army Research Laboratory, AMSRL-SE-RU, Adelphi, MD 20783 USA.

Publisher Item Identifier S 0196-2892(97)03457-8. ultra-wide bandwidths . Unfortunately, this Green's function cannot be expressed in closed form, although each component of the dyadic can be represented as a Sommerfeld integral [15], [24]-[26]. Various numerical and asymptotic algorithms have been developed for the efficient analysis of the Sommerfeld integrals [15], [27]-[29], however none of these are sufficiently general and efficient to be useful for UWB applications. Recently, Chow et al. [30]-[32] have refined the method of complex images to efficiently compute the half-space Green's function, making possible the efficient and accurate computation of UWB-SP scattering from general buried targets [16], [17] (and the complex resonances thereof [18]). Previously we have concentrated on the algorithm's mathematical derivation and numerical implementation [16]-[18], while here we investigate the UWB-SP resonances and scattered fields from realistic targets; in particular, we consider conducting anti-personnel mines, anti-tank mines, and a 55-gallon drum.

Concerning target resonances, Baum [33] introduced the singularity expansion method (SEM) to demonstrate that the late-time transient fields scattered from a target can be expressed compactly in terms of the target's resonant modes. Further, the complex resonant frequencies represent unique discriminants that can be exploited in the context of timedomain ATR. Over the last two decades, researchers have been pursuing SEM-based ATR, through the use of such schemes as Prony 's method [34] and the matrix-pencil method [35], [36]. Such techniques have also been pursued for GPR, with several impressive results reported [5]. However, one would expect that resonance-based identification of buried targets will be very difficult for low- $Q$ targets and/or targets buried in highloss soils. We address this issue by theoretically investigating the late-time resonant frequencies of a realistic buried mine.

Where appropriate, we compare our theoretical calculations with measurements performed using a novel UWB-SP synthetic aperture radar (SAR) which is implemented by placing a bipolar radar on a mobile boom. The fundamental properties of the SAR are described in [37]. The system measures VV, HH, $\mathrm{HV}$, and $\mathrm{VH}$ polarizations and operates in the time domain with an instantaneous bandwidth of approximately 0.1-1.5 $\mathrm{GHz}$, and has an approximate peak radiated power of 150 $\mathrm{kW}$. We have recently used this system to perform UWB-SP SAR measurements at the Yuma Proving Grounds in Yuma, AZ. All our calculations are performed using soil parameters measured from typical Yuma soil, and some of our theoretical results are compared with measured data. 


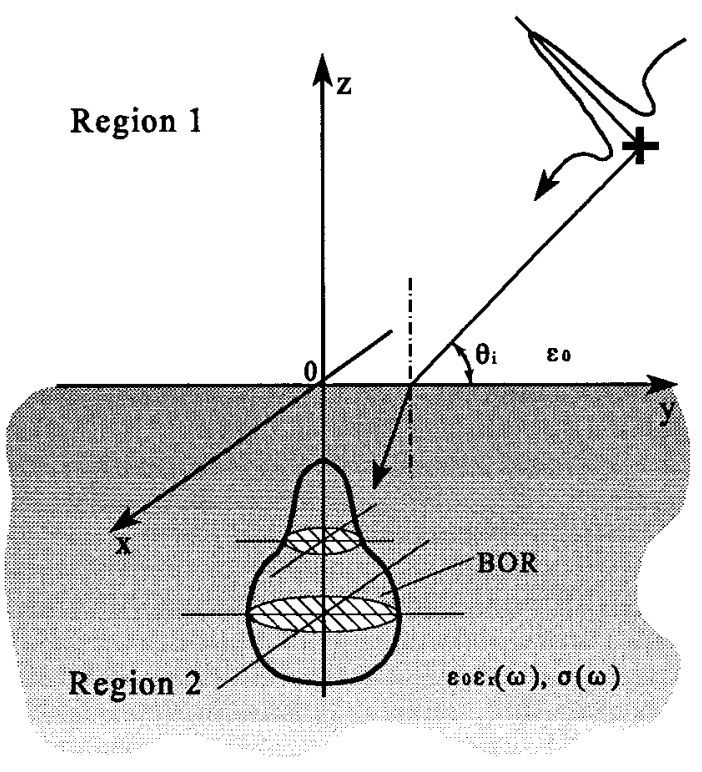

Fig. 1. Schematic of short-pulse plane-wave scattering from a buried perfectly conducting body of revolution.

The remainder of the text is organized as follows. In Section II a brief summary of our numerical algorithm is presented, followed in Section III by calculated results for the scattered fields and resonances of anti-personnel and anti-tank mines, as well as buried canisters. In Section IV we describe the properties of our UWB-SP SAR, and compare measured data with the results of some of our computations. Finally, conclusions are addressed in Section V.

\section{Summary of Numerical Algorithm}

A general perfectly conducting body of revolution (BOR) buried in a lossy, dispersive half space is schematized in Fig. 1. In our algorithm, the target can be placed in (as in Fig. 1) or above the model soil. The latter case is important for military applications, since anti-personnel mines are often placed on the earth's surface. By restricting ourselves to bodies of revolution, the azimuthal variation of the unknown currents on the surface of the target can be expanded in terms of a discrete Fourier basis [17], [18], [38], with subsectional basis functions used to model variation along the generating arc. In the MoM analysis, testing functions [17], [18], [38] with properties similar to the basis functions are used to enforce the boundary condition of vanishing tangential electric field on the target surface, which decouples the problem into an infinite number of matrix equations for the unknown currents-one equation for each azimuthal Fourier component. Only a finite number of Fourier components are necessary to achieve convergence, and therefore a relatively small number of equations are obtained for the two-dimensional currents along the generating arc (the third dimension is accounted for subsequently by summing the various Fourier harmonics [17], [18], [38]). For the scattering problem, inhomogeneous matrix equations are derived for each Fourier component, the driving function of each represented by the azimuthal Fourier transform of the incident tangential electric field on the target surface, evaluated for the discrete Fourier component under consideration [17], [18], [38]. The time-domain scattered fields are synthesized by weighting the frequency-domain scattered fields by the spectrum of the desired incident pulse shape, which is converted to the time domain via Fourier transform. On the other hand, the target resonant frequencies corresponding to a particular azimuthal Fourier component are solved for by setting the driving function (incident field) equal to zero, which yields a homogeneous matrix equation for the modal currents, with nontrivial solutions at the complex frequencies for which the matrix determinant vanishes; thus, by setting the determinant of the matrix to zero, one can solve for the complex resonant frequencies, from which the modal currents can then be computed [18].

The above procedures for computing the scattered fields and complex resonant frequencies of bodies of revolution have been investigated extensively for targets in free space [38]. Our present problem is more complicated because the half-space dyadic Green's function must be expressed in the form of highly oscillatory, slowly convergent Sommerfeld integrals [16]-[18]. Recently Chow [30]-[32] has developed the method of complex images, which represents the spectraldomain reflection coefficient in terms of a sum of exponentials, with parameters found via Prony [34] or matrix-pencil [35], [36] fitting to the exact spectral reflection coefficient. The subsequent Sommerfeld integrals for each term in the exponential sum can be evaluated in closed form via the Weyl identity [39]. The restriction on such a scheme is that the BOR's axis of rotation must be perpendicular to the air-ground interface so that the (half space)-target composite preserves BOR symmetry (see Fig. 1). While this may be a significant restriction for some applications, for military problems (e.g., mines) such a target orientation is often found in practice.

\section{ShORT-PUlSe SCATtering FROM THREE-DIMENSIONAL TARGETS}

We consider below the short-pulse scattered fields and complex resonances of anti-personnel mines, anti-tank mines, and 55-gallon drums placed atop or buried within soil. Such that some of our calculations can be compared with measurements, the electrical parameters of the soil used in our model were measured from soil samples taken from Yuma, AZ, where experiments were performed using an UWB-SP SAR. The frequency-dependent (dispersive) soil parameters were measured with a network analyzer, and consisted of measuring the reflection coefficient from an open-circuit coaxial probe. Measurements were performed as a function of water content, by percentage weight, and are shown in Fig. 2 for the soil sample used in all subsequent scattering and resonance calculations, with results plotted in the form of complex relative permittivity $\epsilon_{r}=\epsilon_{r}^{\prime}-j \epsilon_{r}^{\prime \prime}$.

There are several inherent difficulties in performing material measurements of the type reported in Fig. 2. In particular, the measured complex dielectric constant is a function of the pressure with which the open-circuit coaxial probe is pressed against the soil; in our measurements the probe pressure was increased until the measured complex permittivity stabilized, 


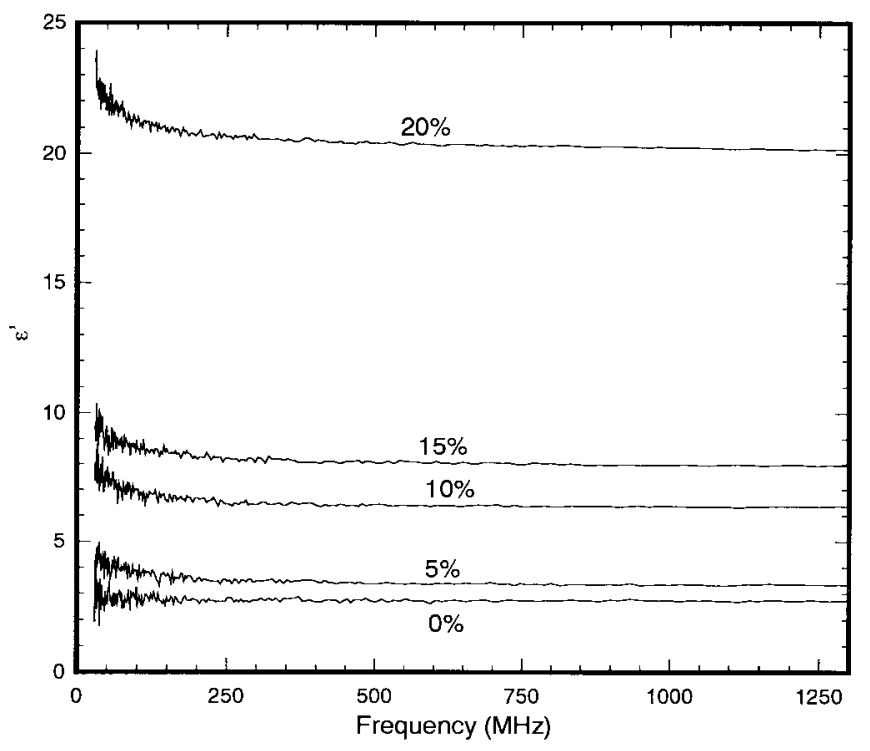

(a)

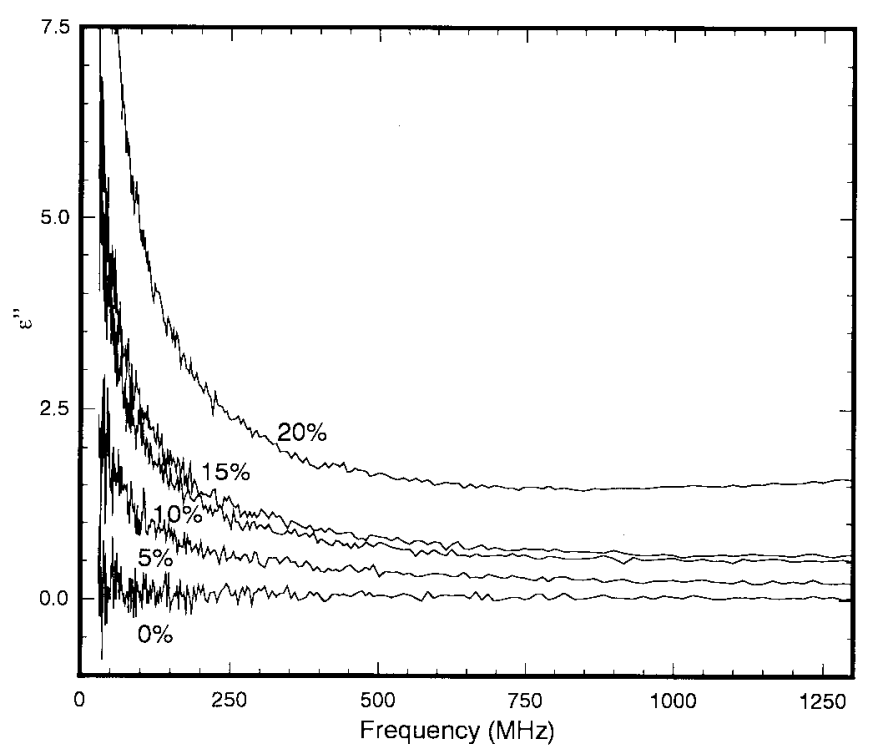

(b)

Fig. 2. Frequency-dependent complex permittivity $\epsilon_{r}=\epsilon_{r}^{\prime}-j \epsilon_{r}^{\prime \prime}$ measured from a soil sample taken from Yuma, AZ. Results are plotted as a function of water content, by weight. (a) $\epsilon_{r}^{\prime}$ (b) $\epsilon_{r}^{\prime \prime}$.

and the measurements were repeated five times and averaged to produce the data in Fig. 2. However, the applied pressure may influence the local percentage of water content in the vicinity of the probe, affecting the measured permittivity. In all subsequent scattering and resonances calculations, the curves corresponding to $5 \%$ water were utilized, in attempt to be consistent with what is typically found in the soils of Yuma, AZ.

\section{A. Buried 55-Gallon Drum}

First, we consider the UWB-SP fields scattered from a buried perfectly conducting 55-gallon drum with axis oriented perpendicular to the air-ground interface. The diameter of the drum is $60 \mathrm{~cm}$, its length is $90 \mathrm{~cm}$, and we consider a case

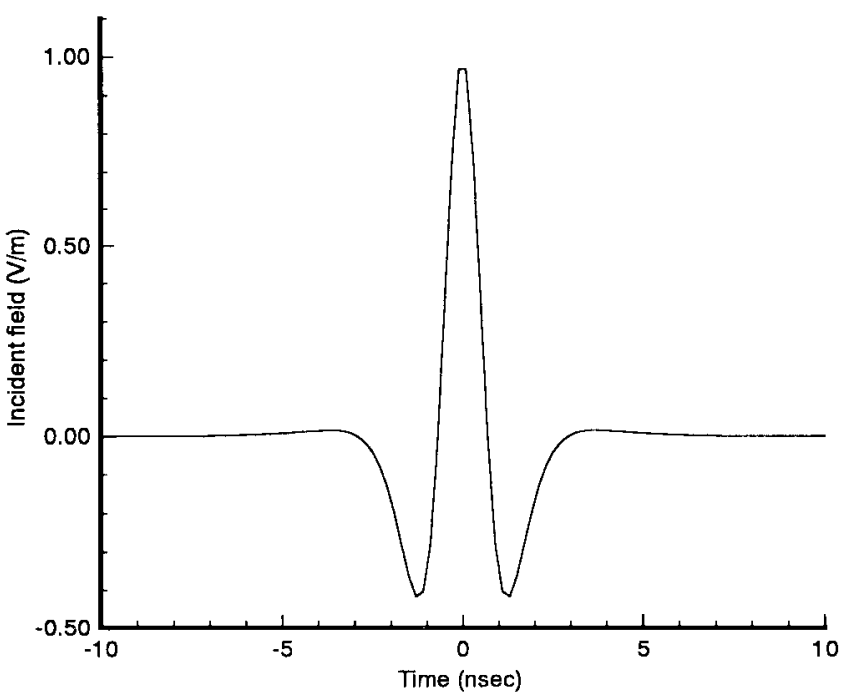

(a)

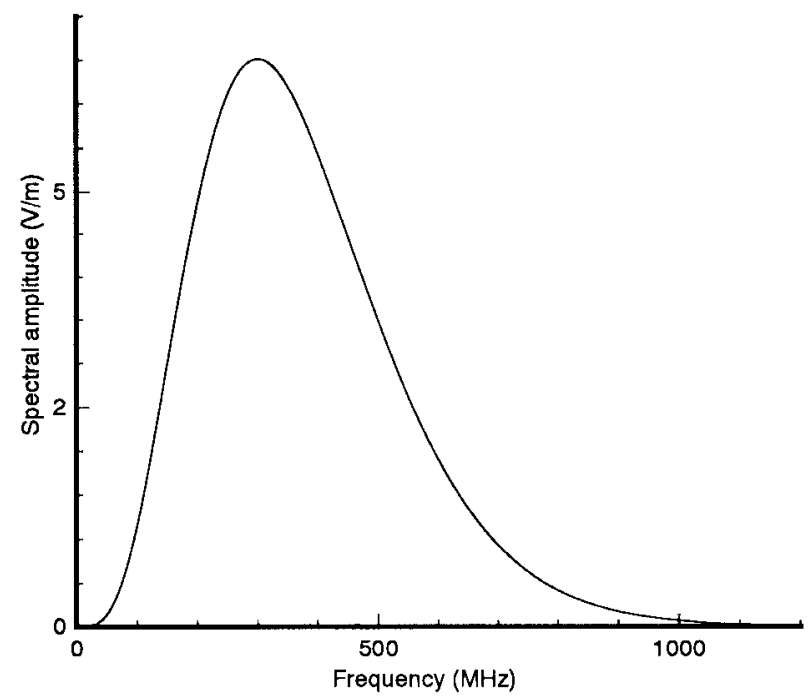

(b)

Fig. 3. Time-domain waveform, and its spectrum, used to describe the shape of the pulsed plane wave considered in subsequent computations. (a) time-domain signal and (b) spectrum.

for which its top surface is buried $30.48 \mathrm{~cm}$ from the airground interface. The time-domain shape of the incident pulsed plane wave is shown in Fig. 3, along with its Fourier spectrum. This waveform is consistent with many current pulsed sources [22], [23], and the low frequencies in the spectrum $(<1.0$ $\mathrm{GHz})$ provide significant ground penetration. In many SAR applications, the incident waveform is near grazing, so we consider an incidence angle of $20^{\circ}$ with respect to the ground, for both $\mathrm{VV}$ and $\mathrm{HH}$ polarization (the cross-polarized scattered fields for bodies of revolution is zero).

The relative power transmitted into the ground for vertically and horizontally polarized plane waves incident at $20^{\circ}$ is shown in Fig. 4. For comparison, in Fig. 4 we also plot the relative transmitted power for a plane wave incident at the approximate Brewster angle at the center frequency $\left(\theta_{B}=28.25^{\circ}\right)$. Note that the Brewster angle is frequency dependent, so Brewster-angle excitation is not possible for all 


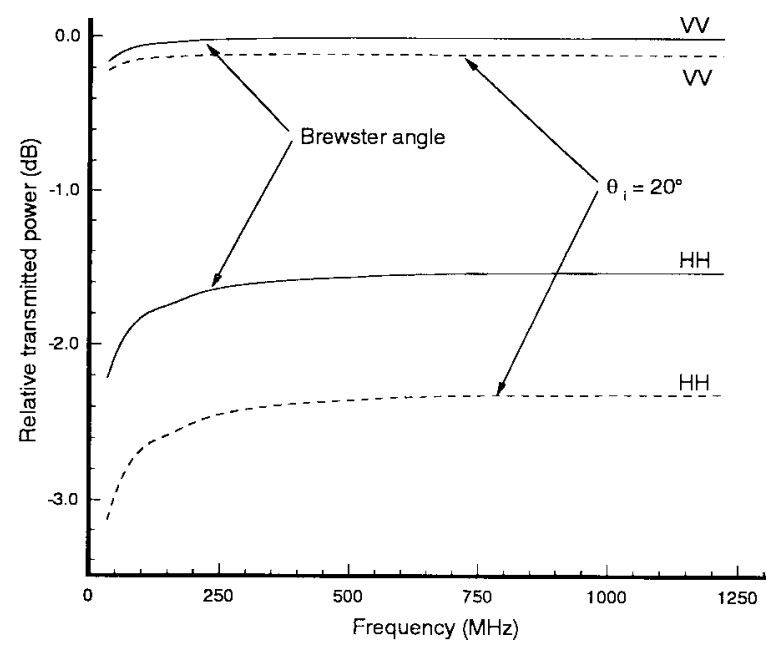

Fig. 4. Relative power transmitted into a lossy halfspace, as a function of frequency, for two angles of incidence (see Fig. 1) and polarization. The halfspace electrical parameters are described in Fig. 2, for the case of $5 \%$ water content.

frequencies in the incident pulse; moreover, for the case of lossy materials, no real Brewster angle exists at any frequency. Fig. 4 shows that the two-way transmission loss for $20^{\circ}$ incidence is approximately $0.1 \mathrm{~dB}$ at the central frequency for VV polarization, and $2.4 \mathrm{~dB}$ for $\mathrm{HH}$ polarization. Further, from the 5\%-water curve in Fig. 2, at the center frequency, there is approximately $4.35 \mathrm{~dB}$ of loss associated with propagation from the air-ground interface to and from the center of the drum. Our time-domain results simultaneously account for loss due to interface effects, propagation loss through the soil, and scattering from the target, and therefore these effects cannot be considered separately; however, from the above discussion, at the pulse's center frequency, the loss from interface and soilpropagation effects combined is approximately $4.45 \mathrm{~dB}$ for VV polarization and $6.75 \mathrm{~dB}$ for $\mathrm{HH}$ polarization.

The normalized VV and $\mathrm{HH}$ time-domain backscattered fields are shown in Fig. 5. Several wavefronts are identified by arrows explained in Fig. 6. Included in Fig. 6 are diffractions from the the top front edge (A), diffraction from the top back edge (B), a reverberation between the front edge and the air-ground interface $(\mathrm{C})$, creeping-wave circumnavigation of the cylinder (D), diffraction from the bottom front edge (E), and multiple diffraction between the top and bottom surface of the drum $(\mathrm{F})$. The $\mathrm{VV}$ and $\mathrm{HH}$ signals differ in several important ways. For example, for VV polarization both edge diffractions " $\mathrm{A}$ " and "B" are strong, while for $\mathrm{HH}$ polarization wavefront " $\mathrm{B}$ " is substantially weaker than "A." The reason for these differences is unclear, due to the complexity of this problem. For example, in reality there is no "front" or "back" edge, but rather a single edge which encircles the entire top surface. Further comparing these results, we see that phenomena " $\mathrm{D}$ " and "E" are excited strongly for VV polarization while they are nearly absent for $\mathrm{HH}$ polarization. Phenomena "D" and "E" occur too closely in time to be individually identified. Therefore, we cannot be sure as to whether this strong wavefront for $\mathrm{VV}$ polarization is due to a creeping wave, edge diffraction, or both. However, for the $20^{\circ}$ angle of incidence, the refracted wave travels at an angle of approximately $30^{\circ}$ relative to the cylinder axis. If the incident wave were to propagate along the axis, no creeping wave is excited at all; therefore we speculate that the creeping wave will be weakly excited for this example in which the incident wave travels almost along the cylinder axis. Interestingly, the multiple edge diffraction, "F," is very similar for both polarizations.

\section{B. Buried Anti-Tank Mine}

There are various anti-personnel and anti-tank mines: some are metal and others dielectric. Their shapes vary widely. In many cases, mines are conducting and disc-like in shape. As examples, we consider two metal mine-like prototypes which simulate a class of anti-personnel and anti-tank mines. Both mines are modeled as perfectly conducting cylinders with axis of rotation perpendicular to the air-ground interface; the antipersonnel mine has a $6.35-\mathrm{cm}$ diameter and $5.08-\mathrm{cm}$ height, while the respective anti-tank mine dimensions are $38.1 \mathrm{~cm}$ and $6.35 \mathrm{~cm}$. We first consider short-pulse scattering from the anti-tank mine, which is usually buried at shallow depths. The incident pulsed plane wave is the same as in Fig. 3. However, we consider incidence at the Brewster angle $\left(\theta_{B}=28.25^{\circ}\right)$, with soil transmission properties described in Fig. 4.

Results are shown in Fig. 7 for the VV and HH transient fields scattered from the anti-tank mine buried at depths of $2.54 \mathrm{~cm}, 7.62 \mathrm{~cm}, 12.7 \mathrm{~cm}$, and $17.8 \mathrm{~cm}(1,3,5$, and $7 \mathrm{in}$, respectively) from the top of the target. The most striking characteristic of these results is the similarity between the VV and $\mathrm{HH}$ fields. Apparently the incident pulse does not have sufficient temporal (spatial) resolution to resolve features on the target, so we do not see the distinctive signatures found in Fig. 5 for the much larger 55-gallon drum. Interestingly, initially the peak scattered signal increases as the target depth increases, in contrast with our anticipation. We attribute this phenomenon to reverberations between the top of the target and the air-ground interface that constructively interfere with wavefronts scattered from the target. As expected, as the target depth is further increased, the peak scattered waveform starts to diminish (the reverberated waveform is attenuated and the temporal overlap of the primary and reverberated wavefronts diminishes).

Fig. 7 does not show an obvious late-time resonant signature associated with the buried anti-tank mine. To explain, we plot in Fig. 8 the complex resonant frequencies of the lowest-order SEM mode for the anti-tank mine considered in Fig. 7. For comparison, the resonant frequency of the lowest-order mode is also plotted for the same target situated in a homogeneous medium of the same electrical properties. Notice that the resonant frequency changes as a function of target depth. In Fig. 9 are plotted the normalized modal currents associated with the mode at each depth. There is a noticeable change in the modal current shape as the target depth is adjusted, with the currents concentrating under the target, nearer the high-dielectric soil, as the mine depth decreases. This latter phenomenon is consistent with the well-known concentration of fields in regions of relatively high dielectric constant. 


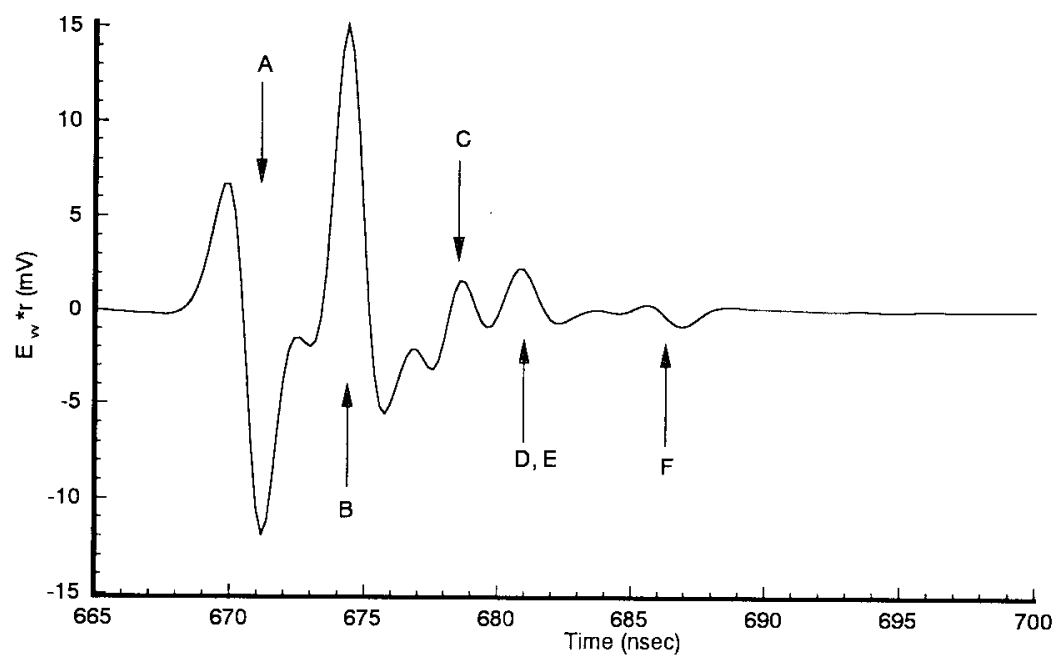

(a)

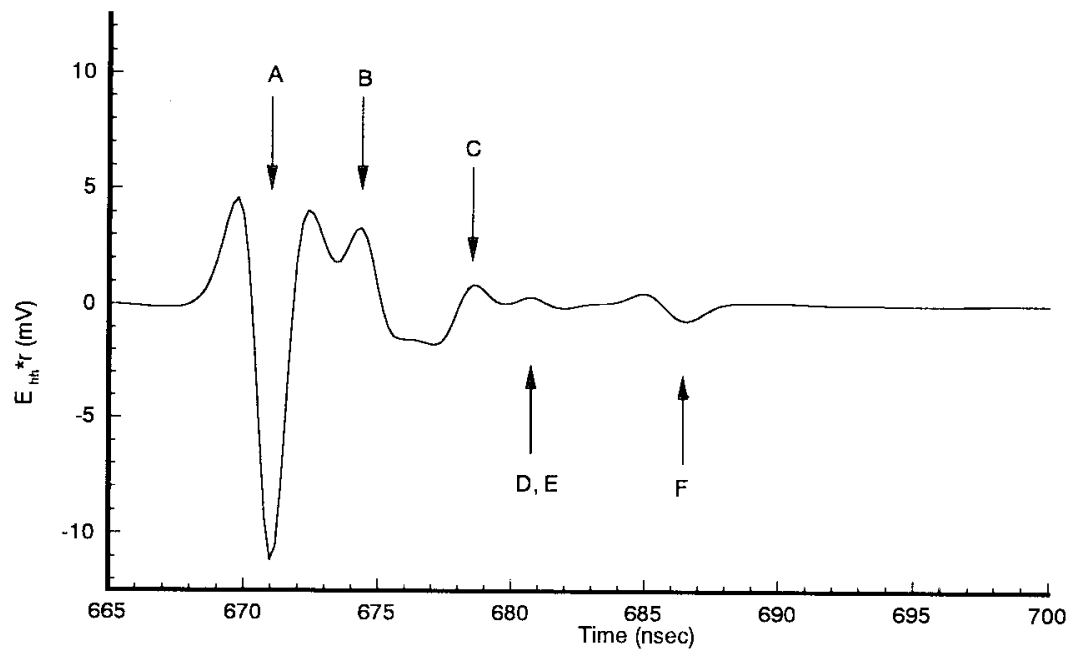

(b)

Fig. 5. Normalized backscattered fields from a buried perfectly conducting cylinder of $60-\mathrm{cm}$ diameter, 90 -cm length, and top surface $30.48 \mathrm{~cm}$ from the air-ground interface; the normalization parameter $r$ is the distance from the surface of the halfspace (on the target axis) to the observer. The incident pulsed plane wave has a temporal shape as in Fig. 3, $20^{\circ}$ angle of incidence, and the soil parameters are for the 5\% case in Fig. 2. (a) VV and (b) HH.
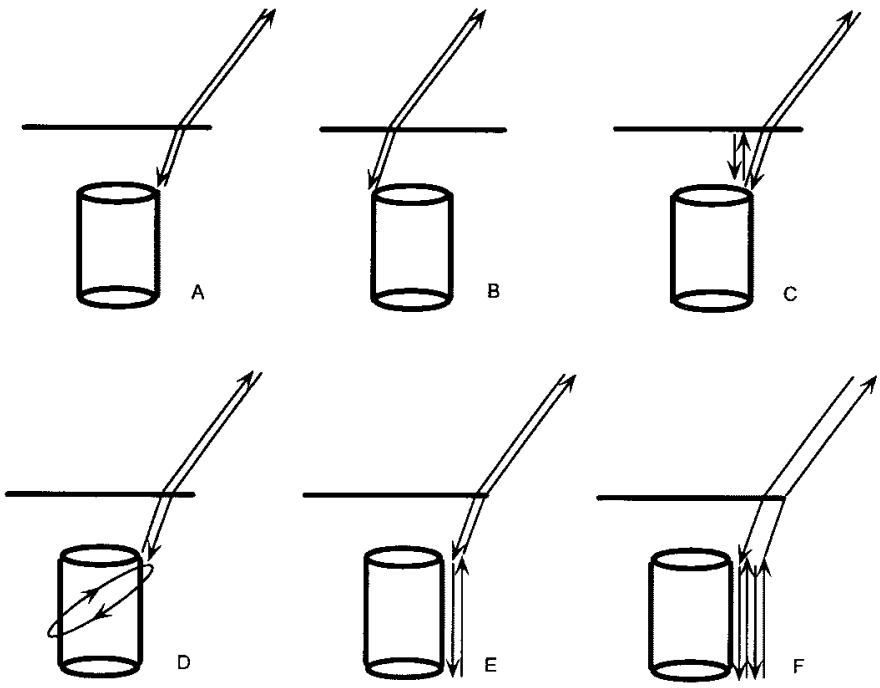

Fig. 6. Schematization of phenomenology characteristic of scattering from a buried cylinder, as considered in Fig. 5. Each scattering mechanism is summarized in the text.
The late-time resonant modes decay with time $t$ as $\exp \left(-\omega_{i} t\right)$, for complex resonant frequency $\omega_{r}+j \omega_{i}$. After $n$ periods the resonant fields decay by $\exp \left(-2 \pi n \omega_{i} / \omega_{r}\right)$. Using the computed resonant frequencies from Fig. 8, after only one oscillation $(n=1)$, the resonant signatures corresponding to depths of $2.54 \mathrm{~cm}, 7.62 \mathrm{~cm}, 12.7 \mathrm{~cm}$, and $17.8 \mathrm{~cm}$ decay by $0.0038,0.0042,0.0041$, and 0.0026 , respectively. These results explain the absence of a discernable resonant signature in Fig. 7, and indicate the extreme difficulty of resonance-based identification for buried targets of the type in Figs. 7-9.

\section{Anti-Personnel Mines}

Typical anti-personnel mines are significantly smaller than anti-tank mines. Additionally, in many cases such mines are placed on the surface of the ground. Obviously, for such situations no ground penetration is required, and we therefore consider higher operating frequencies, such that enhanced down-range resolution can be achieved. In particular, we consider the same waveform in Fig. 3, except its center frequency 


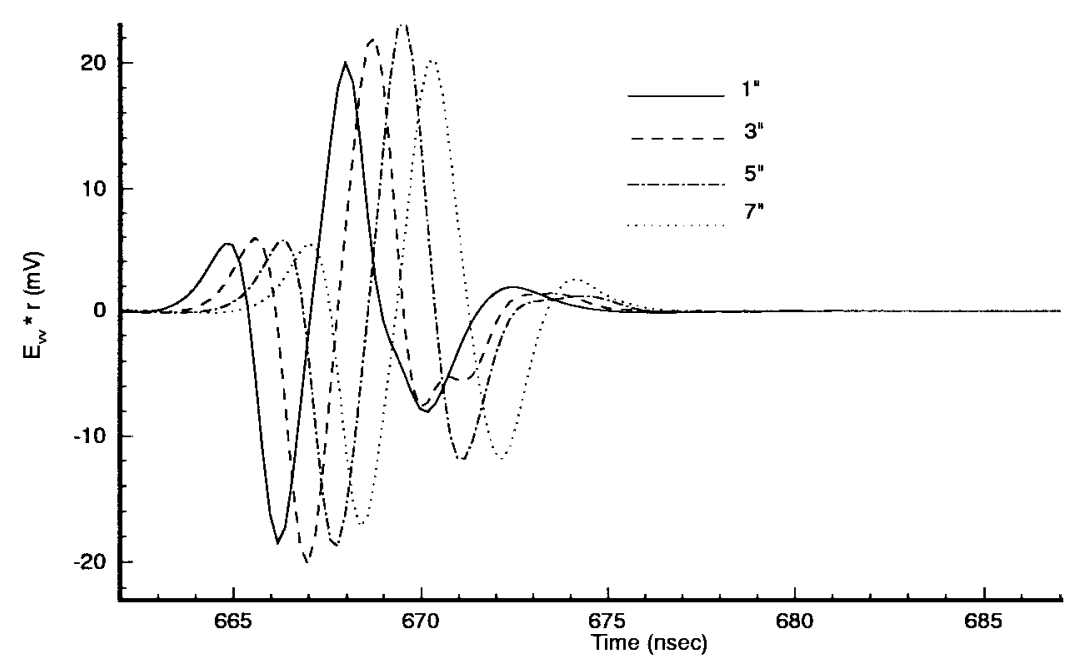

(a)

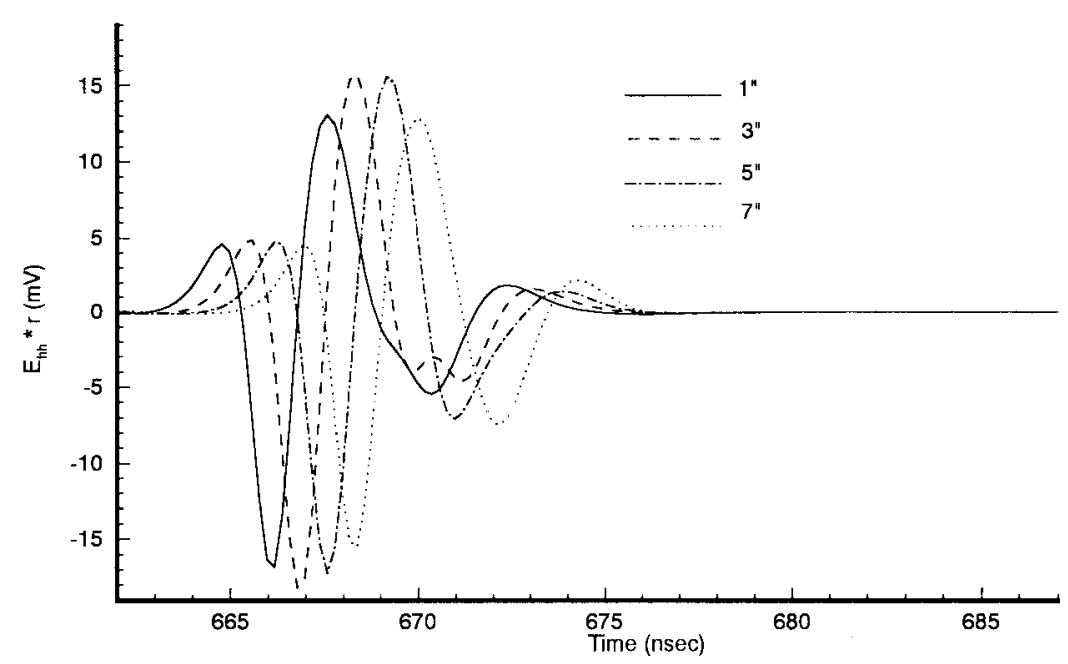

(b)

Fig. 7. Normalized fields backscattered from a model anti-tank mine buried at depths of $2.54 \mathrm{~cm}, 7.62 \mathrm{~cm}, 12.7 \mathrm{~cm}$, and $17.8 \mathrm{~cm}$ from the top of the target. The anti-tank mine is modeled as a perfectly conducting cylinder of diameter $38.1 \mathrm{~cm}$ and height $6.35 \mathrm{~cm}$, with axis perpendicular to the air-ground interface. The pulsed plane wave is incident at the Brewster-angle and incident-pulse shape, soil properties, and normalization are as in Fig. 5. (a) VV and (b) HH.

is moved to $3 \mathrm{GHz}$ (for a total spectrum of approximately $1-10 \mathrm{GHz})$.

We consider a pulsed plane wave incident at $20^{\circ}$ relative to the air-ground interface, and the perfectly conducting antipersonnel mine is placed atop the surface of the Yuma soil (5\% water content). The $\mathrm{VV}$ and $\mathrm{HH}$ polarized scattered fields are shown in Fig. 10, each characterized by two wavefronts. The time interval between the two wavefronts in Fig. 10 corresponds approximately to round-trip propagation over a distance equal to the mine diameter, with account taken for the oblique angle of incidence. Finally, a major difference between the VV and $\mathrm{HH}$ scattered waveforms involves the amplitude of the second wavefront; this issue is addressed further in Section $\mathrm{IV}$, in the context of measured data.

On a numerical note, the electric-field integral equation we use to solve the scattering problem is generally corrupted by artifacts at the internal resonances of a closed target [40], such as that considered in Fig. 10. Due to the high-frequency nature of the incident fields considered in Fig. 10, there are several internal resonances in our spectrum, which affect the scattered fields. This problem has been ameliorated by explicitly enforcing the condition of vanishing electric fields inside the perfectly conducting model mine [40].

\section{UWB-SP SAR MEASUREMENTS}

\section{A. UWB-SP BoomSAR}

The Army Research Laboratory (ARL) has been pursuing the use of UWB-SP SAR's for a number of years to help understand foliage penetrating and ground penetrating radar and develop and qualify suitable wide-band models for predicting performance of proposed systems. These radars provide $1 \mathrm{GHz}$ of instantaneous bandwidth and the full polarization matrix to accomplish this task. The current implementation, hereafter referred to as the BoomSAR, is a mobile extension of the original design that allows data collection over a wide range of clutter and target-in-clutter scenarios. The majority of the hardware is mounted to the basket of a telescoping boom lift 


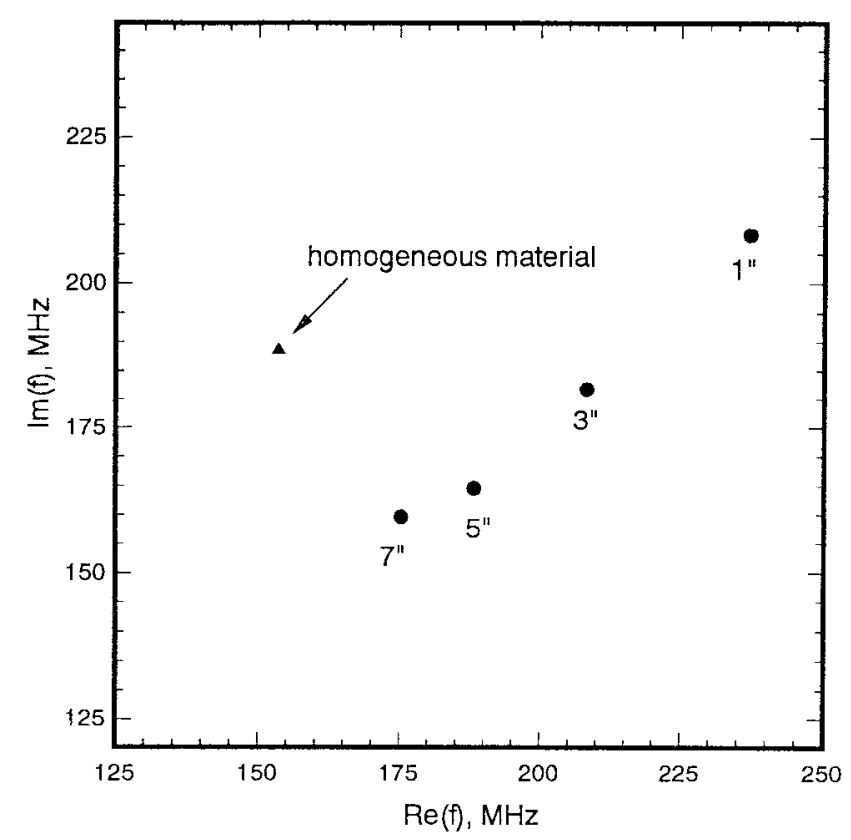

Fig. 8. Complex resonant frequencies of the lowest-order resonant mode for the anti-tank mine considered in Fig. 7.

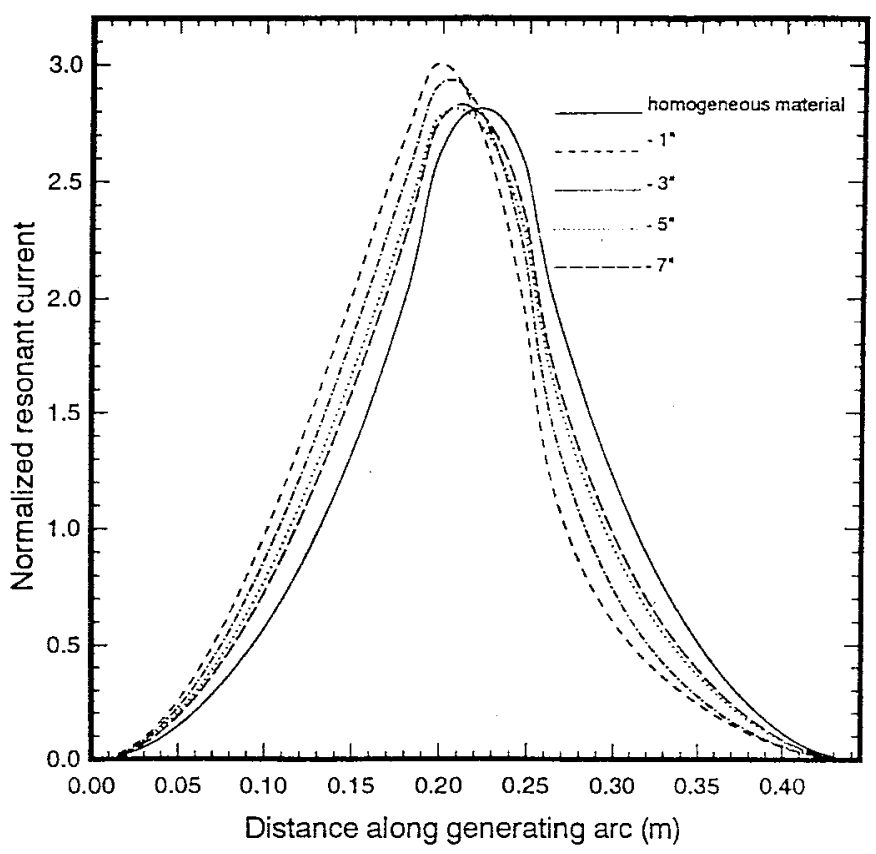

Fig. 9. Normalized modal currents, as a function of target depth, corresponding to the resonant modes in Fig. 8. The currents are normalized such that they integrate to unity when integration is performed along the generating arc of the body of revolution.

capable of moving at approximately $1 \mathrm{~km} / \mathrm{h}$ while the basket is elevated to $45 \mathrm{~m}$. For typical collection geometries, downlook angles to the target vary from $45^{\circ}$ to approximately $10^{\circ}$, depending on the range to the target and the height of the boom.

The radar consists of several major modules. The transmitter is based on a gallium arsenide bulk avalanche semiconductor switch (GaAs BASS). A pair of Power Spectra BASS 02X, pseudo-exponential waveform impulse generators drive the

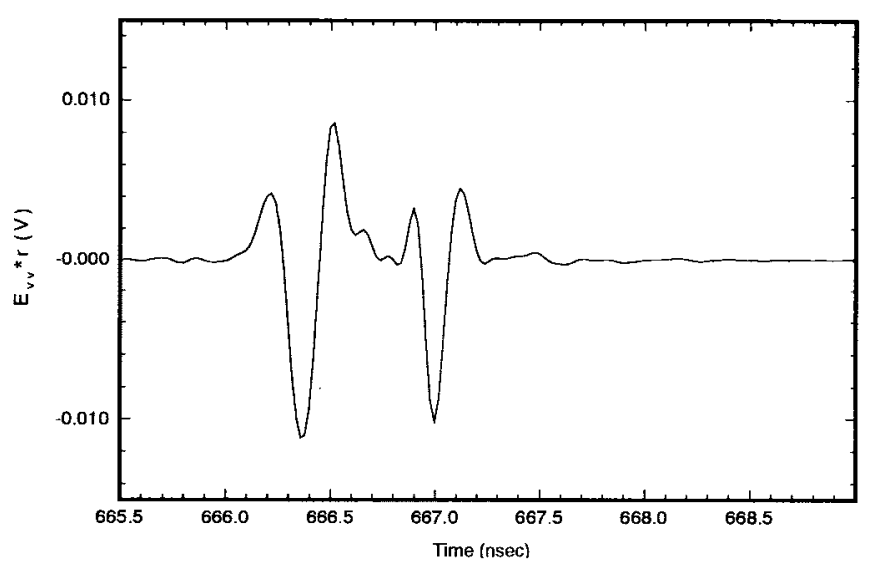

(a)

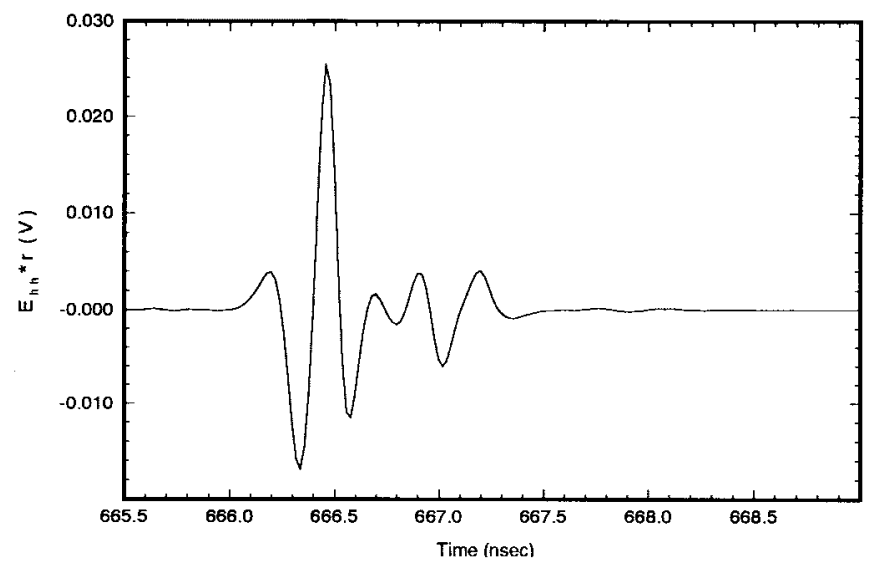

(b)

Fig. 10. Normalized fields backscattered from an anti-personnel mine placed on the surface of Yuma soil (see Fig. 2). The model anti-personnel mine is a perfectly conducting cylinder of $6.35-\mathrm{cm}$ diameter and $5.08-\mathrm{cm}$ height, with axis perpendicular to the air-ground interface. The angle of incidence is $20^{\circ}$, the incident pulse is as in Fig. 3 (with its center frequency shifted to $3 \mathrm{GHz}$ ), and the time-domain fields are normalized as in Fig. 5. (a) VV and (b) HH.

transmit antennas, allowing fast polarization switching by having the processor select which transmitter to enable. The transmit waveform has a 150-ps risetime and approximately a 2-ns falltime with a peak power of approximately $2 \mathrm{MW}$. In the current configuration, the radar produces a burst of 128 pulses of one transmit polarization, followed by a burst of 128 pulses of the opposite polarization, and both receive channels are operated in parallel. There are four antennas: two transmit and two receive, to provide the full polarization matrix $(\mathrm{HH}, \mathrm{HV}$, $\mathrm{VH}, \mathrm{VV}$ ) in a quasimonostatic sense. The antennas are $200 \mathrm{~W}$ open sided, resistively terminated, TEM horns about $2 \mathrm{~m}$ in length with a $0.3-\mathrm{m}$ aperture, and are fed by a wide-bandwidth balun.

With the exception of preamplifier/attenuator assembly, which sets the gain and noise figure for the system, the A/D subsystem acts as the wideband receiver for the radar. The A/D subsystem consists of a pair of Tektronix/Analytek VX2005C, 2-GHz A/D converters, and a stable reference clock. A unique feature of these A/D converters is that they provide, to 10-ps resolution, the time difference between the sample clock and trigger event. With the use of this data, 


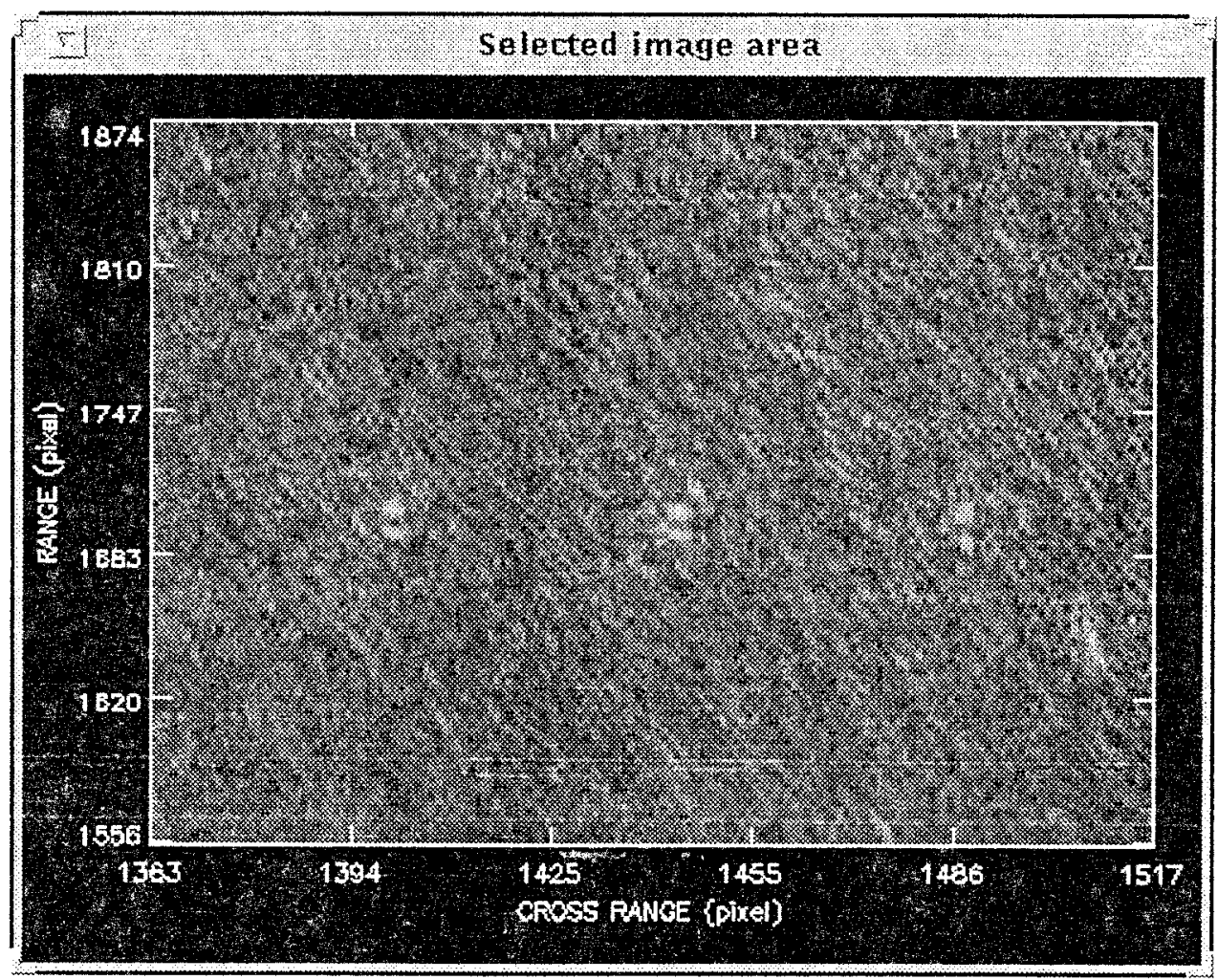

(a)

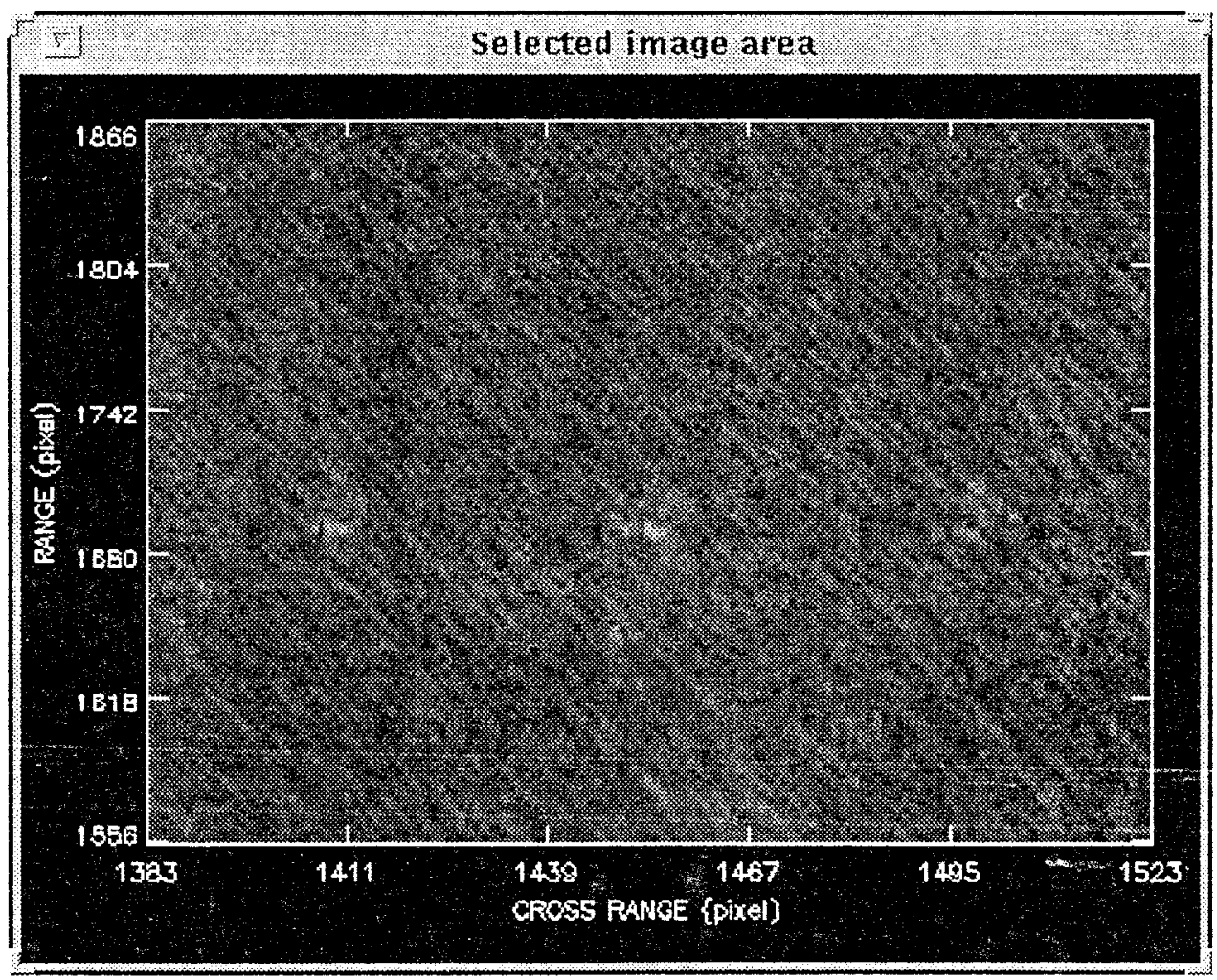

(b)

Fig. 11. Synthetic aperture radar (SAR) image of three surface mines, as measured by an ultra-wideband BoomSAR. The details of the radar and how the image was formed are described in the text. (a) VV and (b) HH. 


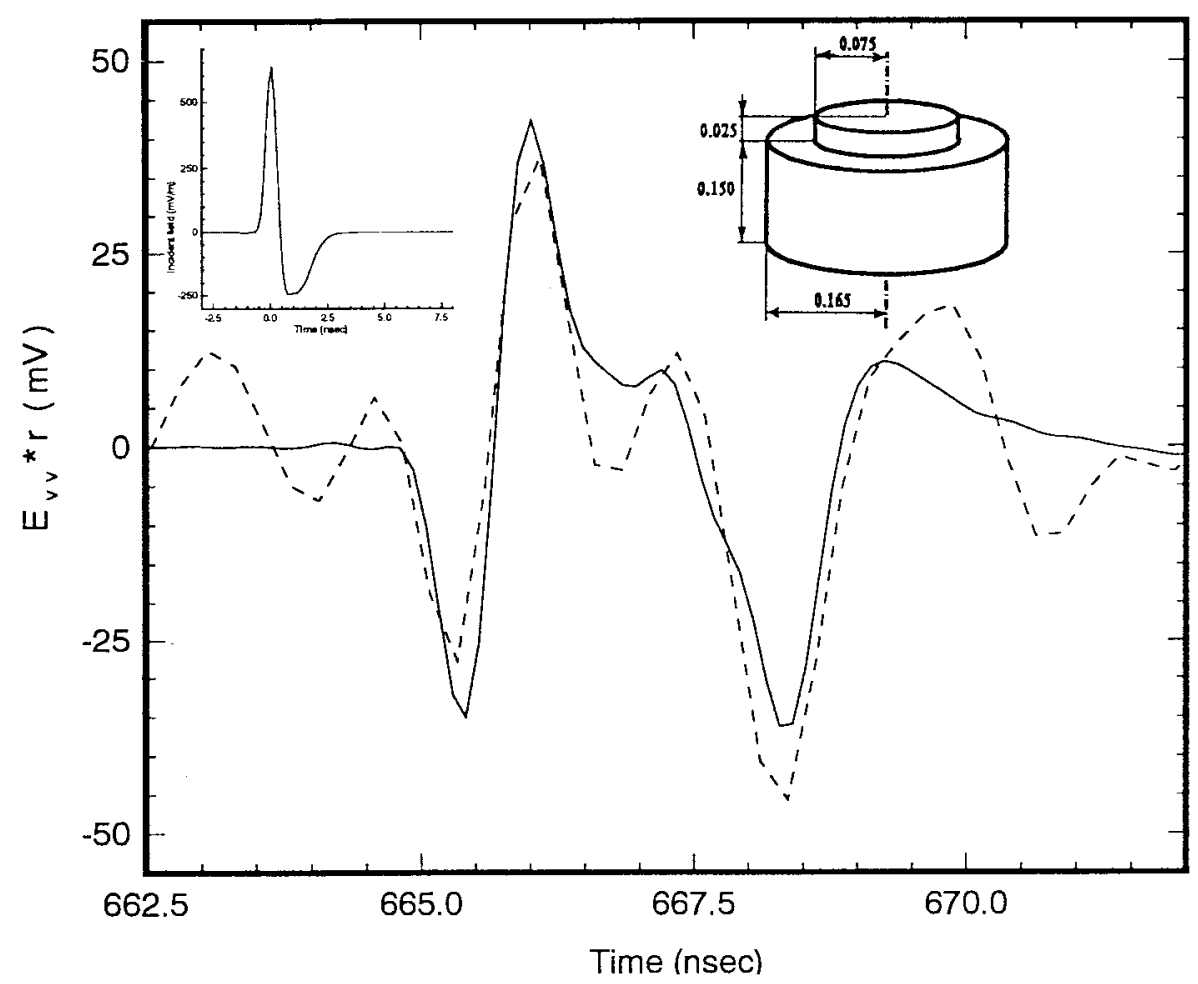

Fig. 12. Comparison between theoretical (solid) and BoomSAR (dashed) scattered fields from a surface anti-tank mine (shown inset). Results are shown for VV polarization and $20^{\circ}$ angle of incidence; the shape of the incident pulse (inset) was measured and is characteristic of the waveform transmitted by the BoomSAR.

subsample time interpolation allows the processor to generate an interleaved record at an equivalent $64-\mathrm{GHz}$ rate, much as would be provided by a sampling oscilloscope. The actual high-frequency response of the radar is defined by the 1100 $\mathrm{MHz}$ analog bandwidth of the A/D converters, while the low frequency response is determined by the $60-\mathrm{MHz}$ response of the antenna/balun assembly.

As in conventional SAR, the BoomSAR is moved along a straight path, and for each position the scattered response is measured. In its simplest embodiment, the bipolar image amplitude $I_{m, n}$ at pixel position $(m, n)$ is given by

$$
I_{m, n}=\sum_{k=1}^{K} s_{k}\left(t=T_{k, m, n}\right)
$$

where $s_{k}(t)$ represents the time-domain scattered field measured at the $k$ th sensor position and $T_{k, m, n}$ is the round-trip time delay between sensor position $k$ and the physical position in the image represented by pixel $(m, n)$. In Section IV$\mathrm{C}$ we use the results of the bipolar image, while for other applications unipolar (magnitude) SAR imagery is generated by Hilbert transforming the bipolar image [37]. The focused image has a resolution of $0.15 \mathrm{~m}$ in the range dimension, and $0.3 \mathrm{~m}$ in the cross-range dimension.

\section{B. Example Measured Results}

We have recently performed an extensive test of our BoomSAR at Yuma Proving Grounds in Yuma, AZ. We present here some relevant results. In particular, we consider surface mines, with geometries similar to that in Fig. 10. However, in those calculations, the center frequency was fixed at $3 \mathrm{GHz}$, whereas the center frequency of our BoomSAR is approximately 500 $\mathrm{MHz}$. For the target sizes to be comparable, we consider a patch of (larger) anti-tank mines situated on the surface of the Yuma soil.

In Fig. 11 are shown BoomSAR measured images (magnitude) of patches of anti-tank mines, for $\mathrm{VV}$ and $\mathrm{HH}$ polarization and $20^{\circ}$ angle of incidence. Interestingly, the signatures of the mines for $\mathrm{VV}$ polarization are characterized by two strong signals, while each of the mines in the $\mathrm{HH}$ image is characterized by only one strong response. This disparity in the $\mathrm{VV}$ and $\mathrm{HH}$ signatures motivated the calculations in Fig. 10. Considering the results in Fig. 10, we see that for VV polarization the model data is characterized by two strong returns, while for the $\mathrm{HH}$ data the second wavefront is substantially smaller than the first. We believe these theoretical results explain the measured contrasts in the $\mathrm{VV}$ and $\mathrm{HH}$ imagery of Fig. 11.

\section{Comparison of Computed and Measured Data}

The particular example considered here is for scattering from the surface anti-tank mine schematized in Fig. 12. Results are presented for $\mathrm{VV}$ polarization, and a $20^{\circ}$ incidence angle is considered, consistent with the data in Fig. 10. In Fig. 12 is shown a comparison between the computed (solid) and measured (dashed) data. The measured data represents the coherent superposition of several sensor positions. Therefore, one would anticipate that the imaging process will cause some blurring in the extracted signature. Further, in the theory we assume plane-wave incidence at a fixed angle and with a particular incident-pulse shape; in practice, the incident 
wave can only be approximated as planar, and the angle of incidence and incident-pulse shape can only be determined approximately. Nevertheless, we see in Fig. 12 that for VV polarization there is excellent agreement between theory and experiment. No effort was made to optimize the agreement between theory and experiment in Fig. 12; i.e., these results are typical of what we have found for several examples.

\section{CONCLUSIONS}

A new Method of Moments (MoM) numerical algorithm has been utilized to examine the UWB-SP fields scattered from and the resonances of several targets buried in and placed atop soil. Results have been presented particularly for soil at Yuma, AZ, with account taken for dispersion and loss. The targets considered were a 55-gallon drum, an anti-tank mine, and an anti-personnel mine. The theoretical results for an antitank mine compared well with measurements performed with a BoomSAR.

Results have only been presented for a small subset of targets and one soil type (albeit a relatively favorable, lowloss soil), but this study further substantiates the difficulty of radar-based detection and identification of buried and surface targets. For example, concerning the resonances of the buried anti-tank mine considered, the low- $Q$ of such resonances, coupled with the soil loss, conspired to produce late-time resonant modes which decay extremely quickly with time, making virtually impossible resonance-based identification for such targets. However, resonant-based detection has proven useful for particular dielectric targets buried in frozen soil [5]. This dichotomy points to the need for modeling-which will yield a priori predictions of GPR performance-to assure that the radar is implemented under appropriate circumstances. It is highly unlikely that GPR will be an effective tool for all soil and target types, but when utilized properly, it can be an effective option.

Fortunately, the need for accurate modeling intersects with recent algorithmic developments, which now make possible the modeling of scattering from and the resonances of realistic (3-D) targets buried in lossy, dispersive soils. In this paper, we have been concerned with UWB-SP radar, which involves incident signals with over $100 \%$ bandwidth. Up until very recently, it was virtually impossible to model the scattering of such waveforms from realistic buried targets, due to the complexity of computing the half-space Green's function (needed for the MoM). However, the recent development of the method of complex images, which efficiently computes the Sommerfeld integrals characteristic of the half-space dyadic Green's function, has been utilized here for several realistic and complicated targets of interest. Future studies will further utilize this algorithm to quantify anticipated radar performance as a function of soil and target type. Additionally, the predicted waveforms from our model can also be utilized in the development of matched filters for target detection.

\section{REFERENCES}

[1] L. Peters, Jr., and J. D. Young, "Applications of subsurface transient radars," in Time-Domain Measurements in Electromagnetics, E. K. Miller, Ed. New York: Van Nostrand Reinhold, 1986.
[2] D. L. Moffatt and R. J. Puskar, "A subsurface electromagnetic pulse radar," Geophys., vol. 41, pp. 506-518, June 1976.

[3] M. E. Bechtel and A. V. Alogn, "Antennas and pulses for a vehicularmounted mine detector," Calspan Corp., Buffalo, NY, Rep. MA-5366E-1, 1974.

[4] D. J. Daniels, D. J. Gunton, and H. F. Scott, "Introduction to subsurface radar," Proc. IEE, vol. 135, pt. F, no. 4, pp. 278-320, Aug. 1988.

[5] L. Peters, J. J. Daniels and J. D. Young, "Ground penetrating radar as a subsurface environmental sensing tool," Proc. IEEE, vol. 82, pp. 1802-1822, Dec. 1994.

[6] R. W. P. King and C. W. Harrison, Jr., "The transmission of electromagnetic waves and pulses into the earth," J. Appl. Phys., vol. 39, pp 4444-4452, Aug. 1968.

[7] J. A. Fuller and J. R. Wait, "Electromagnetic pulse transmission in homogeneous dispersive rock," IEEE Trans. Antennas Propagat., pp. 530-533, July 1972.

[8] G. S. Smith and W. R. Scott, Jr., "A scale model for studying ground penetrating radars," IEEE Trans. Geosci. Remote Sensing, vol. 27, pp. 358-363, July 1989.

[9] N. Osumi and K. Ueno, "Microwave holographic imaging of underground objects," IEEE Trans. Antennas Propagat., vol. AP-33, pp. 152-159, Feb. 1985.

[10] C. Liu and C. Shen, "Numerical simulation of subsurface radar for detecting buried pipes" IEEE Trans. Geosci. Remote Sensing, vol. 29, pp. 795-798, Sept. 1991.

[11] J. M. Bourgeois and G. S. Smith, "A fully three-dimensional simulation of a ground-penetrating radar: FDTD theory compared with experiment," IEEE Trans. Geosci. Remote Sensing, vol. 34, pp. 36-44, Jan. 1996.

[12] P. E. Wannamaker, G. W. Hohmann, and W. A. SanFilipo, "Electromagnetic modeing of three-dimensional bodies in layered earths using integral equations," Geophys., vol. 49, pp. 60-74, Jan. 1984.

[13] H. S. Chang and K. K. Mei, "Scattering of electromagnetic waves by buried and partly buried bodies of revolution," IEEE Trans. Geosci. Remote Sensing, vol. 23, pp. 596-592, 1985.

[14] G. Kristensson and S. Strom, "Scattering from buried inhomogeneities-A general three dimensional formalism," in Proc. 1977 URSI Symp. on Electromagnetic Wave Theory, June 1977.

[15] K. A. Michalski and D. Zheng, "Electromagnetic scattering and radiation by surfaces of arbitrary shape in layered media, Parts I and II," IEEE Trans. Antennas Propagat., vol. 38, pp. 335-352, Mar. 1990.

[16] S. Vitebskiy and L. Carin, "Moment-method modeling of short-pulse scattering from and the resonances of a wire buried inside a lossy, dispersive half space," IEEE Trans. Antennas Propagat., vol. 43, pp. 1303-1312, Nov. 1995.

[17] S. Vitebskiy, K. Sturgess, and L. Carin, "Short-pulse scattering from buried perfectly conducting bodies of revolution," IEEE Trans. Antennas Propagat., vol. 44, pp. 143-151, Feb. 1996.

[18] S. Vitebskiy and L. Carin, "Resonances of perfectly conducting wires and bodies of revolution buried in a lossy, dispersive half space," IEEE Trans. Antennas Propagat., vol. 44, pp. 1575-1583, Dec. 1996.

[19] G. S. Smith and W. R. Scott, "The use of emulsions to represent dielectric materials in electromagnetic scale models," IEEE Trans. Antennas Propagat., vol. 38, pp. 323-334, Mar. 1990.

[20] W. R. Scott and G. S. Smith, "Measured electrical constitutive parameters of soil as functions of frequency and moisture content," IEEE Trans. Geosci. Remote Sensing, vol. 30, pp. 621-623, May 1992.

[21] J. E. Hipp, "Soil electromagnetic parameters as functions of frequency, soil density, and soil moisture," Proc. IEEE, vol. 62, pp. 98-103, Jan. 1974.

[22] H. L. Bertoni, L. Carin, and L. B. Felsen, Eds., Ultra-Wideband, ShortPulse Electromagnetics. New York: Plenum, 1994.

[23] L. Carin and L. B. Felsen, Eds., Ultra-Wideband, Short-Pulse Electromagnetics II. New York: Plenum, 1995.

[24] A. Sommerfeld, "Uber die Ausbreitung der Wellen in der draht Telegraphie," Ann. Phys., vol. 28, pp. 665-736, 1909.

[25] Y. Rahmat-Samii, R. Mittra, and P. Parhami, "Evaluation of Sommerfeld Integrals for lossy half-space problems," Electromagn., vol. 1, no. 1, pp. 1-28, 1981.

[26] E. F. Kuester and D. C. Chang, "Evaluation of Sommerfeld integrals associated with dipole sources above the earth," Univ. Colorado Electromagn. Lab. Sci. Rep. 43, Jan. 1979.

[27] J. R. Wait, "Image theory of a quasistatic magnetic dipole over a dissipative half-space," Electron. Lett., vol. 5, no. 13, pp. 281-282, June 1969.

[28] S. F. Mahmoud and A. D. Metwally, "New image representations for dipoles near a dissipative earth," Radio Sci., vol. 21, pp. 605-616, Nov. 1981. 
[29] I. V. Lindell and E. Alanen, "Exact image theory for the Sommerfeld half-space problem, Part III: General formulation," IEEE Trans. Antennas Propagat., vol. AP-32, no. 10, pp. 1027-1032, Oct. 1984.

[30] Y. L. Chow, J. J. Yang, D. G. Fang, and G. E. Howard, "A closed-form spatial Green's function for the thick microstrip substrate," IEEE Trans. Microwave Theory Tech., vol. 39, pp. 588-562, Mar. 1991.

[31] J. J. Yang, Y. L. Chow, D. G. Fang, "Discrete complex images of a three- dimentional dipole above and within a lossy ground," Proc. Inst. Elec. Eng., vol. 138, pt. H, no. 4, pp. 319-326, Aug. 1991.

[32] R. M. Shubair and Y. L. Chow, "A simple and accurate complex image interpretation of vertical antennas present in contiguous dielectric half spaces," IEEE Trans. Antennas Propagat., vol. 41, pp. 806-812, June 1993.

[33] C. E. Baum, "On the singularity expansion method for the solution of electromagnetic interaction problems," Air Force Weapons Lab, Interaction Notes, Note 88, 1971.

[34] M. Van Blaricum and R. Mittra, "A technique for extracting the poles and residues of a system directly from its transient response," IEEE Trans. Antennas Propagat., vol. AP-23, pp. 777-781, Nov. 1975.

[35] Y. Hua and T. K. Sarkar, "Generalized pencil-of-function method for extracting poles of an EM system from its transient response," IEEE Trans. Antennas Propagat., vol. 37, pp. 229-234, Feb. 1989.

[36] Y. Hua and T. K. Sarkar, "Matrix pencil method for estimating parameters of exponentially damped/undamped sinusoids in noise," IEEE Trans. Acoustics, Speech, Signal Processing, vol. 38, pp. 814-824, May 1990.

[37] M. A. Ressler and J. W. McCorkle, "Evolution of the Army Research Laboratory ultra-wideband test bed," in Ultra-Wideband Short-Pulse Electromagnetics II, L. Carin and L. B. Felsen, Eds. New York: Plenum, 1995, pp. 109-123.

[38] M. G. Andreasen, "Scattering from bodies of revolution," IEEE Trans. Antennas Propagat., vol. AP-13, pp. 303-310, Mar. 1965.

[39] W. C. Chew, Waves and Fields in Inhomogeneous Media. New York: IEEE Press, 1995

[40] F. X. Canning, "Robust use of supplementary conditions for moment method solution near internal resonances," IEEE Trans. Antennas Propagat., vol. 43, pp. 264-269, Mar. 1995.

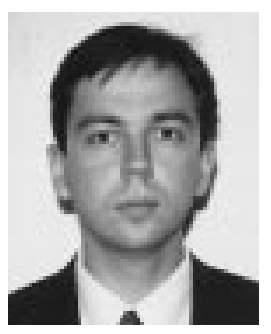

Stanislav Vitebskiy (S'94) received the Dipl. Eng. (honors) degree from the Moscow Institute of Electronics Engineering, Moscow, Russia, in 1992, and the M.S. and Ph.D. degrees, both in electrical engineering, from Polytechnic University, Brooklyn, NY, in 1994 and 1996, respectively.

From 1993 to 1995, he was Graduate Research Assistant with the Department of Electrical Engineering, Polytechnic University. From 1995 to 1996, he was Graduate Research Assistant with the Department of Electrical and Computer Engineering, Duke University, Durham, NC. Currently, he is with Harris Corporation, RF Communications Division, Rochester, NY, working in the wireless communications area. His interests also include numerical modeling in electromagnetics and ground-penetrating radar.

Dr. Vitebskiy is a member of Eta Kappa Nu.
Lawrence Carin (S'85-M'86-SM'96) was born on March 25, 1963 in Washington, DC. He received the B.S., M.S., and Ph.D. degrees in electrical engineering from the University of Maryland, College Park, in 1985, 1986, and 1989 , respectively.

In 1989, he joined the Electrical Engineering Department at Polytechnic University, Brooklyn, NY, as an Assistant Professor, and became an Associate Professor in 1994. In September 1995, he joined the Department of Electrical Engineering, Duke University, Durham, NC, where he is an Associate Professor. His current research interests include quasiplanar transmission lines, short-pulse scattering and propagation, and signal processing.

Dr. Carin is a member of the Tau Beta Pi and Eta Kappa Nu honor societies.

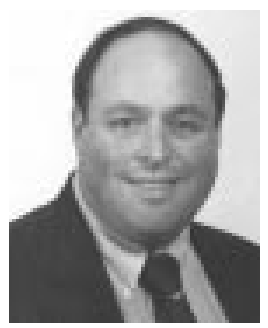

Marc A. Ressler (S'66-M'69) received the B.S degree in electrical engineering from the University of Maryland, College Park, in 1969 and the M.S degree in electrical and computer engineering from the University of Michigan, Ann Arbor, in 1973.

He has been employed by the Army Research Laboratory (ARL) and one of its predecessors, the Harry Diamond Laboratories, since 1969, where he worked on electronic fuzes for rockets, laser simulators and instrumentation systems, adaptive antennas, low-power, stand-alone radar, acoustic, and seismic sensor processors, multi-target tracking for the Installation Security Radar, and a microprocessor-based metering system for the electric power industry (for which a patent was awarded). More recently, he has been involved in battlefield data processing and fusion, and a microwave modular radar system that had applications to both airborne and ground-based systems, including the multi-sensor Elevated Target Acquisition System. He is currently assigned to the Sensors and Electron Devices Directorate of ARL where his area of interest is low-frequency, ultra-wideband radar for foliage and ground penetration applications.

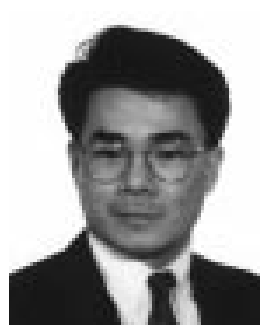

Francis H. Le was born in Saigon, Vietnam, on November 23, 1965. He received the B.S.E.E. degree from the University of South Florida, Tampa, in 1986, and the M.S.E.E. degree from the John Hopkins University, Baltimore, MD, in 1991.

From 1987 to 1993 , he was a part of the design and development team of the Missile Systems Branch, Army Research Laboratory (ARL), to develop analog and digital proximity fuzes for the Patriot missile system. In 1993, he joined the Microwave Branch at ARL to focus on research and development of the UWB (ultra-wideband) SAR (synthetic aperture radar) for FOPEN (foliage penetration) and GPEN (ground penetration). His work covers UWB SAR data reduction, SAR image algorithms development, signal processing, and GUI tools development. 